\title{
Aplicación de la eSalud en el contexto cubano
}

\author{
M. Niurka Vialart Vidal, ${ }^{1}$ María J. Vidal Ledo, ${ }^{1}$ Yanetsys Sarduy Domínguez, ${ }^{1}$ \\ Ariel Delgado Ramos, ${ }^{2}$ Alfredo Rodríguez Díaz, ${ }^{2}$ lleana Fleitas Estévez, ${ }^{3}$ \\ Madelayne Muñoz Morejón, ${ }^{1}$ Xaily Gavilondo Mariño ${ }^{1}$ y Rodolfo Pérez Matar ${ }^{1}$
}

Forma de citar

Vialart Vidal MN, Vidal Ledo MJ, Sarduy Domínguez Y, Delgado Ramos A, Rodríguez Díaz A, Fleitas Estévez I, et al. Aplicación de la eSalud en el contexto cubano. Rev Panam Salud Publica. 2018;42:e19. https://doi.org/10.26633/RPSP.2018.19.

RESUMEN Se exponen los logros y los desafíos que provienen de la aplicación de la eSalud en el contexto cubano para apoyar la salud universal. El Ministerio de Salud Pública definió la Estrategia de Informatización, cuyas líneas de trabajo se sustentan en los componentes declarados en la Estrategia y Plan de Acción sobre eSalud de la Organización Panamericana de la Salud, y en la Politica para la Informatización de la Sociedad, aprobada por el Gobierno cubano. Se especifican las acciones de la estrategia cubana elaboradas para solventar las limitaciones en la infraestructura tecnológica, desarrollar las plataformas, las herramientas y las aplicaciones que requieran los servicios de salud, y estimular en los profesionales las habilidades para emplearlas. Los resultados de la eSalud en Cuba son producto de la aplicación y la evaluación de la estrategia aplicada en el marco del perfeccionamiento del sector de la salud, mediante proyectos como el registro médico electrónico denominado Galen Clínicas, la preparación del capital humano y los recursos de información y educación a distancia de la Red Telemática de Salud Infomed. Estos son modelos de solidaridad y trabajo colaborativo en redes, que han contribuido a la salud universal e impactado positivamente en el sistema de salud, más allá de las fronteras. Los desafíos de la eSalud consisten en el desarrollo de iniciativas en salud móvil, en lo que existe poca experiencia; la telemedicina, cuyo proyecto quedó detenido; la integración e interoperabilidad de las aplicaciones; la sostenibilidad de las soluciones implementadas; la insuficiente infraestructura tecnológica; y el fortalecimiento del marco legal.

Palabras clave Registros electrónicos de salud; telemedicina; cobertura universal; proyectos de tecnologías de información y comunicación; Cuba.

La Organización Mundial de la Salud (OMS) ha planteado nuevas estrategias para afrontar los problemas de la salud en la sociedad de la información y el conocimiento del siglo XXI. Una de estas es la

\footnotetext{
Escuela Nacional de Salud Pública, La Habana, Cuba. Enviar la correspondencia a M. Niurka Vialart Vidal, vialart@infomed.sld.cu
}

llamada eSalud, que consiste en el apoyo que la utilización — con una adecuada relación costo-eficacia y seguridad- de las tecnologías de la información y las comunicaciones (TIC) ofrece a la salud y a los

\footnotetext{
2 Ministerio de Salud Pública, La Habana, Cuba. Organización Panamericana de la Salud, La Habana, Cuba.
}

ámbitos relacionados con ella, incluidos los servicios de atención de salud, la vigilancia y la documentación sanitarias, así como la educación, los conocimientos y las investigaciones en materia de salud $(1,2)$.

La eSalud busca mejorar los flujos de información a través de los medios electrónicos para apoyar los servicios de

Este es un artículo de acceso abierto distribuido bajo los terminos de la licencia Creative Commons Attribution-NonCommercial-NoDerivs 3.0 IGO, que permite su uso, distribución y reproducción en cual quier medio, siempre que el trabajo original se cite de la manera adecuada. No se permiten modificaciones a los artículos ni su uso comercial. Al reproducir un artículo no debe haber ningún indicio de que la OPS o el artículo avalan a una organización o un producto específico. El uso del logo de la OPS no está permitido. Esta leyenda debe conservarse, junto con la URL original del artículo. 
salud y la gestión de los sistemas sanitarios (3). Como señalara la Dra. Carissa Etienne, Directora de la Organización Panamericana de la Salud (OPS), "si se le da un buen uso y se aplica ampliamente, la eSalud puede ser una herramienta estratégica que permita mejorar el acceso, ampliar la cobertura y aumentar la eficiencia financiera de los sistemas de atención de salud" (4).

En el año 2011, la OPS definió la Estrategia y Plan de Acción sobre eSalud para el período 2012-2017 basados en seis componentes: registro médico electrónico (historia clínica electrónica); telemedicina (prestación de servicios de salud mediante las TIC); salud móvil (mSalud: ejercicio de la medicina y la salud pública con apoyo de teléfonos móviles, dispositivos de monitoreo de pacientes y otros dispositivos móviles); aprendizaje con ayuda de las TIC (eLearning, que incluye la formación a distancia); educación continua en TIC (formación de habilidades en TIC para la salud); y estandarización e interoperabilidad (comunicación entre diferentes tecnologías y aplicaciones, mediante el uso de estándares, para una gestión integrada de los sistemas de salud en todos los niveles) $(2,5)$.

En un examen realizado en el 2016, se establecieron cuatro áreas estratégicas: 1) respaldar y promover las políticas públicas en la eSalud; 2) mejorar la salud pública por medio de la eSalud; 3) fomentar y facilitar la colaboración horizontal entre países; y 4) gestionar el conocimiento y la alfabetización digital para mejorar la calidad asistencial, la capacitación y la prevención de enfermedades (6).

La tendencia global, sobre todo en los países desarrollados, es enmarcar la salud en un mercado con múltiples contribuyentes y fuentes de pago, lo que posibilita la informatización del sector, aun cuando en los países en desarrollo no tiene este alcance. No obstante, existen avances en América Latina y el Caribe en la implantación de sistemas y servicios basados en la eSalud, los cuales están vinculados al incremento de la voluntad política y la adopción de medidas relacionadas con el uso de las tecnologías para favorecer la movilización de los recursos necesarios y la inversión en infraestructura (7).

En países como Brasil, Canadá, Chile, Colombia, Costa Rica, El Salvador, Estados Unidos de América, Guatemala, Jamaica, Panamá, Perú y República Dominicana, grupo al que se añade Cuba, están en fase de desarrollo, implementación y ejecución de estrategias nacionales de eSalud. En América Latina y el Caribe se han logrado progresos en este sentido; sin embargo, aún persisten desafíos también en Cuba- sobre los que se trabaja (7).

En este artículo se exponen los logros alcanzados y los desafíos que provienen de la aplicación de la eSalud en el contexto cubano para apoyar la salud universal.

\section{SALUD UNIVERSAL Y eSALUD EN CUBA: EL CONTEXTO}

"Salud universal significa que todas las personas tengan acceso a los sistemas y los servicios de calidad cuando los necesiten, sin sufrir dificultades financieras que los empobrezcan para obtenerlos" (8). Cuba, con una población en el 2016 de 11239224 habitantes (9), cuenta con un Sistema Nacional de Salud (SNS) universal y gratuito constituido por 12409 unidades de servicio (10), cifra que comprende tanto las instituciones médicas como los centros de asistencia social bajo la dirección del Ministerio de Salud Pública (MINSAP). El 18\% de estos servicios radica en la capital del país, de modo que la gran mayoría de las instituciones (82\%) está dispersa por el resto del territorio nacional. El núcleo central del SNS lo conforman 10782 consultorios denominados del médico y la enfermera de la familia, asociados a 451 policlínicos, los cuales conforman las áreas básicas de atención primaria de salud (APS) que cubren $86,2 \%$ del total de las consultas médicas y se hallan vinculados a 150 hospitales (10).

Este conjunto de instituciones requiere de un constante flujo informativo entre todos sus componentes, que favorezca la toma de decisiones oportunas y la satisfacción de las necesidades informativas de las diferentes instancias del gobierno y de sus propios sistemas y servicios (11). Dos factores - la existencia de un único proveedor de servicios de salud para todo el país y la profusión de instituciones de salud con total cobertura geográfica y asistencial- determinan que la introducción de programas nacionales para la informatización de los servicios de salud sea del mayor interés. Por eso, el MINSAP ha mantenido dentro de sus planes de desarrollo las acciones encaminadas a la informatización del sector (figura 1).
La primera estrategia de informatización data de 1997 y se fundamenta en cinco principios rectores: territorialidad interna y externa, planeación metodológica, seguridad, desarrollo en red y sistemas abiertos. En una visita de asesoría realizada por la División de Desarrollo de Sistemas y Servicios de Salud de la OPS, se reconoció esta estrategia como una de las primeras creadas para organizar un trabajo coherente en este sentido (12).

Durante la evaluación de la primera etapa de la estrategia, realizada en el 2001, se notificó la aplicación del Programa de Fortalecimiento de Informática a nivel territorial en $22(75,9 \%)$ de los 29 municipios seleccionados, de los cuales se destacaron tres municipios de Ciudad de La Habana, Cienfuegos y Sancti Spíritus, respectivamente, con flujo informatizado desde las instituciones hasta las instancias de la dirección de salud y los gobiernos provinciales, así como el municipio especial Isla de la Juventud que tenía todas sus instituciones integradas a la red territorial.

El Programa de Telemedicina agrupó 18 instituciones de segundo nivel en el intercambio de imágenes; pero solo la provincia de Santiago de Cuba realizó consultas de segunda opinión entre sus hospitales y el centro de referencia el Hospital Clínico Quirúrgico Hermanos Ameijeiras ubicado en la capital. Otros proyectos vinculados fueron también evaluados de exitosos (12).

La crisis económica que afectó al país a finales del siglo pasado limitó la extensión de las soluciones probadas. Sin embargo, las dificultades impulsaron iniciativas locales y alternativas que durante el período 2002-2016 permitieron mantener los logros y obtener avances en el Registro Informatizado de Salud como plataforma integradora para los sistemas y los servicios de salud, en la esfera de la información científico-técnica y en la Red Telemática de Salud, pero no se pudo llevar a cabo en la extensión de proyectos institucionales, dado lo costoso de su inversión para todo el SNS.

\section{ESTRATEGIA CUBANA DE INFORMATIZACIÓN DE SALUD}

Cuba se encuentra en un proceso de perfeccionamiento de su modelo económico en todos los sectores, en el que se reconoce el papel de la informatización de la sociedad como motor impulsor para su propio desarrollo (13). En este 
FIGURA 1. Hitos de la informatización de la salud en Cuba

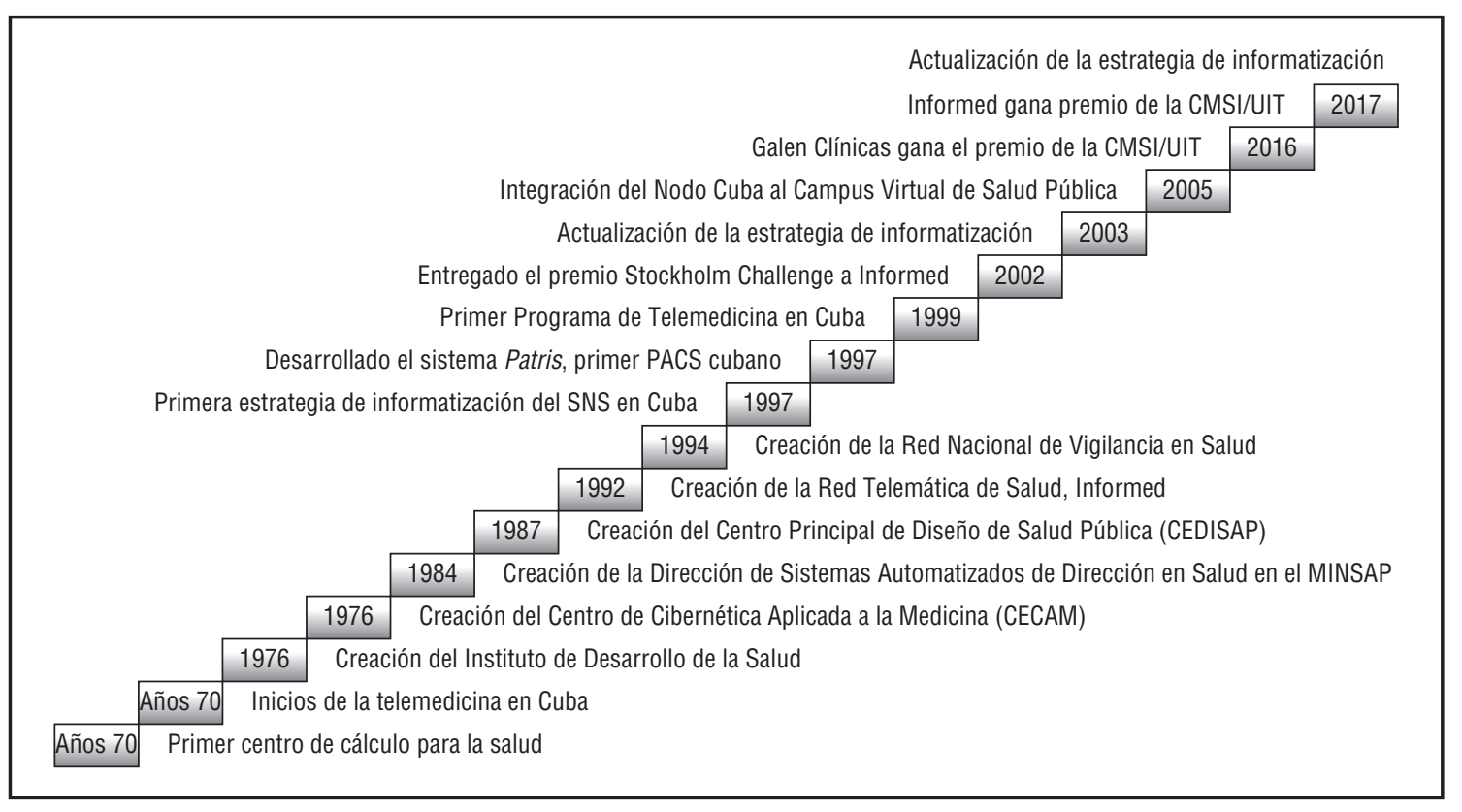

Fuente: Elaboración de los autores.

Notas: CMSI: Cumbre Mundial sobre la Sociedad de la Información; UIT: Unión Internacional de Telecomunicaciones; PACS: Picture Archiving and Comunications System; SNS: Sistema Nacional de Salud; MINSAP: Ministerio de Salud Pública.

contexto, la Dirección Nacional de Informática y Comunicaciones del MINSAP trabaja en la actualización de la base legal y el plan de desarrollo y uso de las TIC hasta el 2030, que para la etapa 20172021 (14) comprende los subsistemas de información en la salud y sus registros, la telemedicina, las redes especializadas y de atención a pacientes, y el sistema empresarial, de logística y aseguramientos. Todo ello se concatena mediante el desarrollo armónico de tres componentes esenciales: la infraestructura (incluida la conectividad), las aplicaciones y los recursos humanos. Esta actualización ha tenido en cuenta la seguridad informática y la ética profesional mediante las normas y las reglas de uso para el acceso y la transmisión, que deben ser cumplidas para garantizar la confidencialidad, la integridad y la disponibilidad de la información (15). Las líneas de trabajo trazadas por la Dirección Nacional de Informática y Comunicaciones integran los componentes de la eSalud bajo los principios establecidos en la estrategia nacional.

\section{Registro médico electrónico}

Los registros médicos electrónicos forman parte del Registro Informatizado de Salud, integrado al Sistema de
Información para la Salud (SISalud), y se ubican en la "nube" de la Red Telemática de Salud Infomed. Además de mostrar los indicadores de salud requeridos, estos registros contienen la historia clínica digital (HCD) única del paciente, identificada por el número de identidad del ciudadano; para revisar ambas informaciones existen niveles de acceso según el tipo de usuario (16-18).

En este componente se han diseñado diversas aplicaciones, la más exitosa es el programa Galen Clínicas, desarrollado por la Empresa Cubana de Soluciones Informáticas, SOFTEL (http://www. softel.cu/), premiado por la Unión Internacional de Telecomunicaciones en la categoría de eSalud en el 2016 (http:/ / www.softel.cu/portal/showNovedad/ ?id=4028908253aacf230153e7f66fe70053). Esta aplicación es la base del Sistema de Información Hospitalaria y abarca los registros médicos, la gestión de medios diagnósticos, consultas y hospitalización, y la emisión de informes de resultados; también garantiza el intercambio de referencias y contrarreferencias entre los niveles de atención, así como la generación de las estadísticas requeridas.

El sistema se ha ido perfeccionando y se implementa de manera modular, según el nivel de atención y la complejidad de cada institución objeto de informatización. De acuerdo con el cronograma establecido para su introducción, se explota ya en $11(91,7 \%)$ institutos de investigación, 53 (35,3\%) hospitales, $100 \%$ de los bancos de sangre territoriales y se encuentra a prueba en 3 clínicas de atención internacional y 2 policlínicos (instituciones de salud del primer nivel de atención) (18, 19). La incorporación del sistema es aún insuficiente en el país, aunque las brigadas médicas cubanas lo emplean como parte de la cooperación internacional en instituciones de Angola, Brasil, Ecuador, Sudáfrica y Venezuela con resultados satisfactorios.

\section{Telemedicina y mSalud}

La telemedicina y la mSalud constituyen retos para el SNS, como una necesidad tecnológica de los servicios de salud (20). Las primeras experiencias nacionales en telemedicina datan de la década de 1970, cuando se incursionó en los sistemas para consultas a distancia. En el período 1998-2001 se implementó el Programa de Telemedicina a escala nacional $(21,22)$. El centro de referencia para el funcionamiento del telediagnóstico es el Hospital Clínico Quirúrgico Hermanos Ameijeiras, de la capital, que cubre todas las especialidades médicas. 
Se realizaron intercambios diagnósticos y consultas de segunda opinión entre profesionales de instituciones de segundo y tercer nivel de atención (22), pero aún no es una práctica generalizada la consulta a través de la telemedicina, y el sistema se ha limitado a la transmisión y el intercambio de imágenes entre instituciones. No obstante, este empleo ha permitido optimizar el uso de los recursos tecnológicos, disminuir los gastos y extender los servicios. Algunos factores de índole financiera impidieron su expansión a las áreas rurales donde se esperaba que tuviera un impacto socioeconómico significativo.

El desarrollo de varios sistemas cubanos - Patris, Imagis y Cassandrapermitió automatizar la transmisión de imágenes y el flujo de información en radiología, cardiología y tomografía axial computarizada, respectivamente, así como el intercambio necesario para las interconsultas (23-25). El sistema Cassandra continúa en explotación en las instituciones cubanas y también en algunas extranjeras como parte de la cooperación internacional en los países ya mencionados (25). Otra de las experiencias exitosas de la telemedicina, reconocida por la OPS por su carácter innovador, es el proyecto de neurociencias, destinado a la detección temprana de la pérdida auditiva en niños, que ha tenido un gran impacto social (26).

Las experiencias en mSalud son mucho más limitadas, aun cuando se reconoce su importancia como vía alternativa que amplía las posibilidades de comunicación entre los profesionales y con toda la población, y que es coherente con la tendencia internacional de ofrecer servicios de salud cada vez más personalizados (27). La incursión en este campo se ha enfocado en dos direcciones: apoyar la docencia, pues se reconoce la utilidad de los dispositivos móviles para la gestión de entornos personales de conocimiento y divulgar información de salud. En este último sentido se destacan, en particular, los servicios de mensajería para el enfrentamiento de la tuberculosis y la promoción de salud de la denominada Línea de Apoyo a las Personas Viviendo con VIH/sida, ambos con suscripción voluntaria y gratuita $(28,29)$.

Asimismo, se promueve investigar y determinar esferas de aplicación mediante la ejecución de proyectos coordinados entre la Escuela Nacional de Salud Pública (ENSAP) y el Centro
Nacional de Prevención de las Infecciones de Transmisión Sexual, el Virus de la Inmunodeficiencia Humana $y$ el Sida (ITS/VIH/sida), y de proyectos relacionados con el seguimiento de los pacientes crónicos a medida que la adquisición de la tecnología lo permita (28).

\section{Experiencias en eLearning}

La preparación de los recursos humanos en todas sus formas ha constituido parte de las estrategias de informatización para la salud. Existen experiencias en modelos de educación continua, desarrollados por las cátedras de la Universidad Virtual de Salud (UVS) en las provincias del país, por la ENSAP y por el Nodo Cuba del Campus Virtual de Salud Pública (https://cuba.campusvirtualsp.org) $(30,31)$.

La UVS tuvo sus antecedentes en los modelos y las plataformas diseñados por varias universidades de Ciencias Médicas; en el año 2000, se integraron en un portal con un aula virtual soportada en la plataforma Moodle, la clínica virtual y el repositorio para la docencia y la investigación. En este portal se encuentran numerosos cursos y se enlaza la red de aulas virtuales de las universidades y los centros docentes y asistenciales del SNS que ofertan recursos de autoaprendizaje y de capacitación, entre otras modalidades docentes (30). El empleo de eLearning ha beneficiado aproximadamente a más de 5000 profesionales de la salud, pero mantiene el reto de extender su uso a la formación de pregrado y con ello incrementar el acceso a la educación en red y a formas innovadoras de enseñanza (31-33).

Cuba integró el Campus Virtual de Salud Pública en el año 2005. El Nodo Cuba está conformado por una red de instituciones y personas que crean, comparten y colaboran en los procesos educativos para alcanzar buenas prácticas en la salud pública. En este se han compartido — con alcance regionalcursos virtuales, recursos educativos abiertos que se enlazan al repositorio, contribuciones al espacio de la clínica virtual, discusiones diagnósticas, consultas a expertos, aspectos de clínica patológica y estudios de casos, y se ha apoyado el trabajo científico-docenteasistencial para impulsar la educación permanente y las redes especializadas (cuadro 1).

\section{Educación continua en TIC para la salud}

Séror plantea que el sistema cubano de salud ofrece un modelo que muestra cómo un portal nacional de información de salud contribuye a la integración de sus elementos, incluidas la investigación, la educación y la prestación de servicios, así como el intercambio internacional de productos médicos (34).

La Licenciatura en Sistemas de Información en Salud es una carrera universitaria de perfil amplio diseñada a partir de un riguroso proyecto de investigación y acción; se imparte en todas las universidades de ciencias médicas del país y cuenta con varias modalidades de posgrado. Entre el 2003 y el 2016 han egresado 4759 profesionales que laboran en información y tecnologías para los servicios de registros médicos, estadísticas sanitarias, bibliotecología médica e información científico-técnica, informática para la salud y seguridad informática (35).

La Red Telemática de Salud (www.sld. cu) es el portal de la salud cubana, conocido en el país y el mundo como Infomed; surgió a finales de 1992 como un proyecto del Centro Nacional de Información de Ciencias Médicas para mitigar las restricciones para adquirir y diseminar la información científico-técnica que impuso la difícil situación económica que sufría el país desde finales de 1989 (36). Su papel en la educación continua, como centro de posgrado en ciencias médicas y en particular en las TIC, es innegable, por lo que ha devenido en una fortaleza para la educación médica a través de sus plataformas de eLearning y los servicios de valor agregado.

Infomed constituye la infraestructura de las comunicaciones sobre la que se sustenta la informatización del SNS y soporta el desarrollo de numerosos contenidos y servicios de toda la red de la salud. Actualmente interconecta 1327 instituciones de todas las provincias, brinda servicios profesionales $\mathrm{y}$ conforma la "nube" del SNS, con 16 nodos provinciales y más de 300000 usuarios mediante un nodo nacional con alcance internacional (36). Infomed es reconocido como un proyecto innovador y socializador al servicio de la salud pública cubana, por lo que la Unión Internacional de Telecomunicaciones de las Naciones Unidas le otorgó el premio en la categoría de eSalud en la Cumbre 
CUADRO 1. Contribución académica al Campus Virtual de Salud Pública del Nodo Cuba

\begin{tabular}{|c|c|c|c|}
\hline Modalidad académica & Temas impartidos y recursos & Fecha de las ediciones & Número de egresados ${ }^{a}$ \\
\hline & & 2007 & 12 \\
\hline & Promoción de salud & 2008 & 24 \\
\hline & & 2010 & 28 \\
\hline \multirow[t]{4}{*}{ Diplomados } & Promoción de salud en el contexto de la asistencia primaria de salud & 2012 & 17 \\
\hline & & 2015 & 23 \\
\hline & Farmacoepidemiología & 2013 & 14 \\
\hline & & 2015 & 24 \\
\hline \multirow[t]{9}{*}{ Cursos } & Farmacovigilancia & 2014 & 32 \\
\hline & & 2015 & 28 \\
\hline & Técnicas inmunoenzimáticas & 2011 & 17 \\
\hline & & 2012 & 19 \\
\hline & Prevención de la enfermedad meningocócica & 2011 & 21 \\
\hline & & 2012 & 12 \\
\hline & Elementos esenciales para el trabajo en la atención primaria de salud & 2014 & 31 \\
\hline & Alfabetización informacional & 2015 & 10 \\
\hline & Curso de autoaprendizaje sobre recursos educativos abiertos & 2017 & Autoaprendizaje \\
\hline \multirow{2}{*}{ Recursos educativos abiertos } & Recursos educativos generales & $2005-2017$ & 372 \\
\hline & Grabaciones de teleconferencias de enfermería & 2010-2017 & 68 Grabaciones \\
\hline
\end{tabular}

Fuente: Elaboración de los autores.

a Parte de los egresados son originarios de países que se han beneficiado de estas intervenciones educativas: Argentina, Bolivia, Brasil, Chile, Colombia, Cuba, Ecuador, El Salvador, España, Guatemala, México, Nicaragua, Panamá, Paraguay, Perú, Uruguay, Venezuela.

Mundial de la Sociedad de la Información en junio del 2017 (37).

En la universalización del conocimiento en la salud, también se destaca el Centro Virtual de Convenciones de Salud (Cencomed, www.cencomed.sld.cu), que facilita la promoción y el desarrollo de eventos científicos en la modalidad virtual, y el acceso a los profesionales del mundo interesados en participar.

Uno de los servicios más relevantes que brinda la red es la Biblioteca Virtual de Salud (BVS, http:/ /bvscuba.sld.cu), que garantiza un espacio para la integración de las principales fuentes de información. Este servicio, que se inserta en el Sistema de Información Científico-Técnica, ofrece acceso a las bases de datos cubanas y foráneas, la colección de libros de autores cubanos, los enlaces a 53 revistas de la Editorial Ciencias Médicas y 31 sitios temáticos sobre la salud, al repositorio de tesis de doctorado, boletines y otros recursos de gran importancia para la gestión de la información y el conocimiento. El Sistema de Información Científico-Técnica cubano es reconocido por las más importantes revistas científicas internacionales dedicadas a la Internet y la salud, al estar sostenido por criterios de colaboración y participación, más que por su infraestructura tecnológica (36).

Cuba lidera un grupo de redes en las Américas, entre las que se encuentran la
Red de Enfermería Informática, la Red de Enfermería en Salud Infantil, y la Red de Género y Salud Colectiva, que emplean las TIC como alternativa de comunicación y educación continua, difunden información y coordinan acciones de capacitación y proyectos entre varios países (29).

\section{Estandarización e interoperabilidad}

El intercambio de información entre todos los componentes del Sistema de Salud exige la implementación de estándares que garanticen la interoperabilidad. El Grupo de Arquitectura para el Desarrollo de Soluciones Informáticas en Salud se ocupa de estos aspectos. Se adoptaron los estándares internacionales recomendados para la representación de datos clínicos, diagnósticos y procedimientos, de soporte tecnológico y comunicaciones, así como los de identificación, contenido, estructura y lenguajes, dirigidos al diseño del registro computarizado de pacientes, que incluye los registros dentales (38).

Se trabaja, además, en la actualización de la base legal y la definición de regulaciones y otras especificaciones que permitan la compatibilidad entre las tecnologías y las aplicaciones en el sistema de salud cubano, de manera que se garantice su interoperabilidad y su funcionamiento seguro, cohesionado y armónico.

\section{CONSIDERACIONES FINALES}

\section{Avances, insuficiencias y desafíos}

El desarrollo de la eSalud en Cuba ha transitado por diferentes etapas, influidas por las condiciones económicas del país. A pesar de las limitaciones, la informatización del sector se ha mantenido como una prioridad para el SNS. En consonancia con las políticas del país, el MINSAP se encuentra en fase de actualización y revitalización de su estrategia nacional de informatización.

Los proyectos de la eSalud cubana son modelos avanzados de solidaridad, en los que se comparten información y conocimiento mediante el trabajo colaborativo de las redes temáticas y los servicios de salud. Los recursos educativos y de información contenidos en la UVS y la BVS, la educación a distancia, las posibilidades de organizar eventos científicos virtuales, entre otros, han contribuido a la formación de los recursos humanos. No obstante, aún persisten insuficiencias de índole económico-financiera que restringen el desarrollo tecnológico requerido en el sector y hay limitaciones en el marco legal y regulatorio y en el establecimiento de los estándares que faciliten 
CUADRO 2. Lecciones aprendidas durante la introducción de la eSalud en Cuba

\begin{tabular}{|c|c|c|}
\hline Componente & Dificultades & Soluciones \\
\hline Registro médico electrónico & $\begin{array}{l}\text { - Restricciones para la adquisición del } \\
\text { equipamiento por problemas financieros } \\
\text { - Dificultad en el diseño e implantación del sistema } \\
\text { de registro electrónico para la atención de salud al } \\
\text { ciudadano } \\
\text { - Insuficiente base legal en la cobertura de los } \\
\text { registros electrónicos }\end{array}$ & $\begin{array}{l}\text { - El Estado priorizó la informatización del sector, con el aporte del presupuesto y la } \\
\text { obtención de otras fuentes de financiamiento } \\
\text { - Se contrató a terceros para el desarrollo de la historia clínica digital (HCD), como parte } \\
\text { del sistema de informatización hospitalaria } \\
\text { - Se amplió el concepto de HCD, como un único registro de salud para el ciudadano, } \\
\text { independientemente del nivel de atención } \\
\text { - Se validaron los registros propuestos y el programa Galen Clínicas se extendió de } \\
\text { forma progresiva en las instituciones asistenciales }\end{array}$ \\
\hline Telemedicina & $\begin{array}{l}\text { - Insuficiente desarrollo tecnológico } \\
\text { - No se realizan consultas de segunda opinión a } \\
\text { través de la telemedicina }\end{array}$ & $\begin{array}{l}\text { - Se aplicaron los estándares tecnológicos y de conectividad requeridos para este proceso } \\
\text { - Se implantó el repositorio nacional de imágenes médicas y el servicio de telemedicina } \\
\text { en la "nube" } \\
\text { - Se desarrollaron soluciones tecnológicas y estrategias de perfeccionamiento del capital } \\
\text { humano para integrarse al sistema de interconsultas y consultas de segunda opinión }\end{array}$ \\
\hline mSalud & $\begin{array}{l}\text { - Uso insuficiente de dispositivos móviles para la } \\
\text { salud y poco desarrollo de aplicaciones }\end{array}$ & $\begin{array}{l}\text { - Se incentivó la investigación conceptual y la determinación de posibles esferas de } \\
\text { actuación en el ámbito nacional, a medida que la adquisición de la tecnología lo } \\
\text { permitiera } \\
\text { - Se ejecutaron soluciones informáticas para móviles asociadas a la salud }\end{array}$ \\
\hline eLearning & $\begin{array}{l}\text { - Necesidades crecientes de utilización de } \\
\text { ambientes virtuales para el proceso enseñanza- } \\
\text { aprendizaje }\end{array}$ & $\begin{array}{l}\text { - Se realizó el proyecto de la Universidad Virtual de Salud, para dar soporte al modelo de } \\
\text { Universalización de la enseñanza que propicia la educación permanente de los } \\
\text { profesionales de la salud desde sus puestos de trabajo, así como la formación de los } \\
\text { recursos humanos, bajo un modelo de educación a distancia }\end{array}$ \\
\hline $\begin{array}{l}\text { Educación continua en } \\
\text { tecnologías de la } \\
\text { información y las } \\
\text { comunicaciones (TIC) }\end{array}$ & $\begin{array}{l}\text { - Insuficiente conocimiento de las potencialidades } \\
\text { que ofrecen las TIC en el desarrollo de la } \\
\text { educación permanente } \\
\text { - Dificultades en la obtención de materiales } \\
\text { impresos }\end{array}$ & $\begin{array}{l}\text { - Se insertó la asignatura de Informática en los programas de estudio de las carreras de } \\
\text { la salud, que abarca, entre otras temáticas, las TIC } \\
\text { - Se implementó el proyecto de alfabetización informacional que se extiende a todo el } \\
\text { país mediante la Red Telemática de Salud Infomed } \\
\text { - Se estableció la estrategia de digitalizar las revistas científicas } \\
\text { - Todas las revistas cubanas de ciencias médicas pasaron a edición digital } \\
\text { - Se creó la Biblioteca Virtual de Salud y el Centro Virtual de Convenciones de Salud }\end{array}$ \\
\hline $\begin{array}{l}\text { Estandarización e } \\
\text { interoperabilidad }\end{array}$ & - Ausencia de estándares actualizados & $\begin{array}{l}\text { - Se definieron los estándares } \\
\text { - Todos los proyectos ejecutados son estandarizados e interoperables }\end{array}$ \\
\hline
\end{tabular}

Fuente: Elaboración de los autores.

el intercambio de información. Estos factores coartan los procesos de interconsultas y consultas de segunda opinión, los registros médicos y, en particular, la extensión a todo el país de la HCD única para el ciudadano.

Para llegar al nivel de desarrollo alcanzado se han tenido que sortear muchas dificultades, cuyas lecciones aprendidas permitieron establecer las soluciones que han tenido un impacto positivo en la salud pública (cuadro 2). El alcance de estas acciones no se limita al ámbito nacional: han tenido también un impacto positivo en la obtención de importantes resultados de salud en otros países del mundo con los que Cuba colabora y, de

esta forma, en la consecución de la salud universal.

Sin embargo, a pesar de este esfuerzo, aún los resultados en la telemedicina, la mSalud y las HCD son insuficientes y se debe ganar experiencia en los servicios que se ofrecen directamente a los pacientes. Estos son desafíos que debe enfrentar la implementación de la estrategia de informatización de salud integral para el período 2017-2021, que está encaminada a la mejora continua de los servicios de la salud y al avance hacia una sociedad más justa, informada y equitativa.

Agradecimientos. Los autores agradecen a la Escuela Nacional de Salud
Pública, al Ministerio de Salud Pública de Cuba y la Organización Panamericana de la Salud/Organización Mundial de la Salud por sus valiosos aportes en los análisis críticos y los juicios expresados en el presente artículo.

\section{Conflicto de intereses. Ninguno.}

Declaración. Las opiniones expresadas en este manuscrito son responsabilidad de los autores y no reflejan necesariamente los criterios ni la política de la Revista Panamericana de Salud Pública/Pan American Journal of Public Health o de la Organización Panamericana de la Salud.

\section{REFERENCIAS}

1. Organización Panamericana de la Salud, Organización Mundial de la Salud. Estrategia y plan de acción sobre eSalud. 148 sesión del Comité Ejecutivo; 2011 junio 20-21. Washington, DC: OPS; 2011. (Documento CE148/73). Disponible en: http://www.paho.org/ict4health/index. php?option $=$ com_docman\&task $=$ doc view\&gid=7\&Itemid=260 Acceso el 10 de febrero de 2017.

2. Organización Panamericana de la Salud, Organización Mundial de la Salud.
Estrategia y plan de acción sobre eSalud (2012-2017). 51. ${ }^{\circ}$ Consejo Directivo de la OPS, 63. ${ }^{a}$ sesión del Comité Regional de la OMS para las Américas; 2011 septiembre 26-30; Washington, DC: OPS; 2011 (Documento CD51/13). Disponible en: 
http:/ / new.paho.org/hq/index.php?option=com_docman\&task=doc_download\&gid=14573\&Itemid Acceso el 10 de febrero de 2017.

3. Organización Mundial de la Salud, Unión Internacional de Telecomunicaciones. Conjunto de herramientas para una estrategia de eSalud nacional. Ginebra: OMS; 2012.

4. Etienne C. La eSalud: aprovechar la tecnología en el camino hacia la cobertura universal de salud. Rev Panam Salud Publica. 2014;35(5/6):317-9. Disponible en: http:/ / www.scielosp.org/pdf/rpsp/v35n5-6/01. pdf Acceso el 10 de febrero de 2017.

5. World Health Organization, Global Observatory for eHealth. Atlas of eHealth country profiles: the use of eHealth in support of universal health coverage: based on the findings of the third global survey on eHealth 2015. Geneva: WHO; 2016. Disponible en: http://apps.who.int/iris/ bitstream / 10665 / 204523/1 / 978924 1565219_eng.pdf?ua=1 Acceso el 10 de febrero de 2017.

6. Organización Panamericana de la Salud, Organización Mundial de la Salud. Estrategia y plan de acción sobre eSalud: examen de mitad de período. $55 .{ }^{\circ}$ Consejo Directivo, 68. ${ }^{a}$ sesión del Comité Regional de la OMS para las Américas; 2016 septiembre 26-30; Washington, DC: OPS; 2016. (Documento CD55/INF/12). Disponible en: http://www.paho.org/hq/index.php?option=com_docman\&task=doc_download\&gid=35692\&Itemid=270\&lang=es Acceso el 10 de septiembre de 2017.

7. Novillo-Ortiz D, D'Agostino M, BecerraPosada F. El rol de la OPS/OMS en el desarrollo de capacidad en eSalud en las Américas: análisis del período 2011-2015. Rev Panam Salud Publica. 2016;40(2):85-9. Disponible en: http://www.scielosp.org/ scielo.php?script=sci_abstract\&pid$=$ S1020-49892016000800085\&lng $=$ pt\&nr$\mathrm{m}=$ iso\&tlng=es Acceso el 10 de febrero de 2017.

8. Morales Ojeda R. Cobertura universal de salud. Experiencia cubana. Convención Internacional Cuba Salud; 2015 abril 21-22; La Habana; 2015. Disponible en: http:// www.cubadebate.cu/opinion/2015/04 $/ 21 /$ ministro-de-salud-roberto-morales-ojeda-expone-experiencia-de-cuba-encobertura-universal/\#.WiGJum5xnIU Acceso el 10 de septiembre de 2017.

9. Oficina Nacional de Estadística de Cuba. Anuario Estadístico de Cuba: población 2016. La Habana: ONEC; 2017. Disponible en: http://www.one.cu/aec2016/03\% 20Poblacion.pdf Acceso el 10 de febrero de 2017.

10. Oficina Nacional de Estadística de Cuba. Anuario Estadístico de Cuba: salud pública 2016. La Habana: ONEC; 2017. Disponible en: http://www.one.cu/aec2016/19\%20 Salud\%20Publica.pdf Acceso el 10 de febrero de 2017.

11. Delgado A, Vidal M. Informática en la salud pública cubana. Rev Cubana Salud Publica. 2006;32(3). Disponible en: http:/ / scielo.sld.cu/scielo.php?script=sci_arttext\&pid=S0864-34662006000300015\&lng=es Acceso el 18 de marzo de 2017.
12. Vidal Ledo M. Primera Estrategia para la informatización del sector de la Salud Pública Cubana. Una propuesta para el desarrollo. La Habana: Editorial Ciencias Médicas; 2007. Disponible en: http:// www.bvs.sld.cu/libros/prim_estrategia_ inform/completo.pdf Acceso el 18 de marzo de 2017.

13. Partido Comunista de Cuba. Actualización de los Lineamientos de la Política Económica y Social del Partido y la Revolución para el Período 2016-2021. La Habana: Editora Política; 2016. Disponible en: http://www.granma.cu/file/pdf/gaceta/01Folleto.Lineamientos-4.pdf Acceso el 14 de abril de 2017.

14. Ministerio de Salud Pública. Plan de desarrollo y uso de las Tecnologías de la Información y Comunicaciones del Sistema Nacional de Salud 2017-2021. Rev Infodir. 2017;25:133-57. Disponible en: http:/ / revinfodir.sld.cu/index.php/infodir/article/view/432/512 Acceso el 16 de septiembre de 2017

15. Ministerio de Comunicaciones. Reglamento de seguridad para las tecnologías de la información. Resolución N. ${ }^{\circ}$ 127/07. La Habana: Gac Oficial Repub Cuba. 2007; (7)30:57. Disponible en: http://www.mincom.gob.cu/sites/default/files/marcoregulatorio/ 1346872659054 _R\% 20 127-07\%20Reglamen to \% 20de \% 20 Seguridad\%20Informatica.pdf Acceso el 20 de octubre de 2017.

16. Cabrera Arribas YM, González Alfonso YE, Cruz Águila CL. El proceso de prueba del proyecto Sistema de Información para la Salud (SISalud). Ser Cient Univ Cienc Informaticas. 2008;1(2). Disponible en: http://publicaciones.uci.cu/index.php/ SC/article/view/28/29 Acceso el 4 de marzo de 2017.

17. Cabrera Hernández M, Delgado Ramos A Sánchez Rodríguez A, Acuña Sánchez A, Barrios A, Hernández Laborde $\mathrm{A}$, et al. Plataforma para la administración, procesamiento y transmisión de la información en el Sistema de Salud: SISALUD. VII Congreso Internacional de Informática en la Salud; 2009 febrero 9-13; La Habana; Dirección Nacional de Informática en Salud; 2009. Disponible en: http://www.informatica2009.sld.cu/Members/mirnacabrera/ plataforma-para-la-administracionprocesamiento-y-transmision-de-lainformacion-en-el-sistema-deSaludsisalud/ Acceso el 4 de marzo de 2017.

18. Rodríguez Díaz A, García González G, Barthelemy Aguiar K. Proyecto de informatización en el Sistema Nacional de Salud. Enfoques hacia la dirección en salud. Rev Infodir. 2013;(16). Disponible en: http:/ / revinfodir.sld.cu/index.php/infodir/article/view/8/12 Acceso el 16 de septiembre de 2017 .

19. Ministerio de Salud Pública. Anuario Estadístico de Salud. La Habana: Dirección de Registros Médicos y Estadísticas de Salud; 2016. Pp 129.

20. Puig Meneses Y. Consejo de Ministros aprueba políticas para la informatización de la sociedad y para otros importantes sectores. Cubadebate; 2017 marzo 3. Disponible en: http://www.cubadebate. cu/noticias / 2017/03/03/consejo-deministros-aprueba-politicas-para-lainformatizacion-de-la-sociedad-y-otrosimportantes-sectores/ Acceso el 10 de marzo de 2017.

21. Novillo-Ortiz D, ed. Definición de indicadores para proyectos de Telemedicina como herramienta para la reducción de las inequidades en salud: documento de análisis y resultados de una comunidad de prácticas. Washington, DC: Organización Panamericana de la Salud. 2016. Disponible en: http://iris.paho.org/xmlui/handle/ $123456789 / 28563$ Acceso el 10 de febrero de 2017.

22. Manrique JE. Salud-e y Telemedicina en Cuba: logros, planes y retos pendientes. Memorias del II Seminario Regional sobre Salud-e y Telemedicina en América Latina y el Caribe; 2011 julio 26-27; Caracas: Red Pan Amazónica de Ciencia, Tecnología e Innovación en Salud; 2011.

23. Ronda D, Ferrer O, Álvarez NA. IMAGIS: sistema para la transmisión de imágenes médicas multimodales. Memorias II Congreso Latinoamericano de Ingeniería Biomédica; 2001 mayo 23-25; La Habana; Sociedad Cubana de Bioingeniería; 2001. Disponible en http://www.sld.cu/eventos / habana2001/arrepdf/00190.pdf Acceso el 10 de Marzo de 2017.

24. Cruz Martínez I, González Oliva A, Machado Acuña F. Consideraciones sobre la implementación del IMAGIS para la interpretación de imágenes diagnósticas digitales con fines docentes en tecnología de la salud. MEDISAN (Santiago de Cuba). 2013;17(7):2075-8.

25. Guzmán Díaz C, Aguilar V, Bárbaro D. Sistema para el almacenamiento y transmisión de imágenes médicas, versión 3.0. RCIM. 2014;6(1):17-23. Disponible en: http:/ / scielo.sld.cu/scielo.php?script=sci_abstract\&pid=S1684-18592014000 $100003 \& \operatorname{lng}=$ es\&nrm=iso\&tlng=es Acceso el 10 de marzo de 2017.

26. Novillo-Ortiz D, ed. La eSalud en la Región de las Américas: derribando las barreras a la implementación. Resultados de la Tercera Encuesta Global de eSalud de la Organización Mundial de la Salud. Washington, DC: Organización Panamericana de la Salud; 2016.

27. Rodríguez Blanco S, Almeida Gómez J, Cruz Hernández J, Martínez Ávila D, Guerra P, Carlos J, et al. Relación médico paciente y la eSalud. Rev Cubana Invest Biomed. 2013;32(4):411-20. Disponible en: http:/ / scielo.sld.cu/scielo.php?script=sci_abstract\&pid=S0864-03002 $013000400004 \& \operatorname{lng}=\mathrm{es} \& n r m=i s o \& \operatorname{lng}=e s$ Acceso el 28 de febrero de 2017.

28. Gavilondo X, Vialart MN. Salud Móvil: retos y perspectivas de aplicación en Cuba. Rev Cubana Enferm. 2016;32(1):98-106. Disponible en: http://scielo.sld.cu/pdf/ enf/v32n1/enf11116.pdf Acceso el 28 de febrero de 2017.

29. Vialart Vidal N. Alternativas de comunicación para la Cobertura Universal de Salud en las Redes Internacionales de Enfermería. Rev Cubana Enferm. 2015;30(4). Disponible en: http://www.revenfermeria.sld.cu/index.php/enf/article/view/648/109 Acceso el 28 de febrero de 2017. 
30. Zacca González G, Diego Olite FM, Martínez Hernández GM, Vidal Ledo M, Nolla Cao NE, Rodríguez Castellanos L. Manual Metodológico de la UVS. La Habana: Editorial Ciencias Médicas; 2013. Disponible en: http://www.uvs.sld.cu/ sites/default/files/usuarios/aduvs/manual_metodologico_completo.pdf Acceso el 22 de marzo de 2017.

31. Alfonso Sánchez I. Educación a distancia. ACIMED. 2003;11(1). Disponible en: http:/ / www.bvs.sld.cu/revistas/aci/vol11_1_03/ aci02103.htm Acceso el 6 de marzo de 2017.

32. Sánchez RI, Ponjuán G. Diseño de un modelo de gestión de conocimiento para entornos virtuales de aprendizaje en salud. Rev Cuba Inf Cienc Salud. 2016;27(2):13853. Disponible en: http://scieloprueba. sld.cu/scielo.php?script=sci_abstract \& pid=S2307-21132016000200003\&lng=es\& nrm=iso\&tlng=es Acceso el 10 de marzo de 2017.

33. Alfonso Sánchez I. La Universidad Virtual de Salud. Sistema complejo. Rev Infodir. 2013; 17:38-43. Disponible en: http:// www.revinfodir.sld.cu/index.php / infodir/article/view/36/40 Acceso el 18 de marzo de 2017.

34. Séror AC. A case analysis of INFOMED: the Cuban National Health Care Telecommunications Network and Portal. J Med Internet Res. 2006;8(1):e1. Disponible en: http:/ / www.jmir.org/2006 /1/e1/ Acceso el 10 de marzo de 2017.

35. Vidal Ledo M, Díaz Montes de Oca F, Fuentes Gil Z, Armenteros Vera I, Araña Pérez AB, Castañeda Abascal I. Calidad del proceso de enseñanza-aprendizaje en la carrera de Tecnología de la Salud en Sistemas de Información en Salud. Rev Educ Med Sup. 2011;25(4):466-85. Disponible en: http:/ / scielo.sld.cu/scielo.php?script=sci_ arttext\&pid=S0864-21412011000400008\& lng=es\&nrm=iso\&tlng=es Acceso el 16 de septiembre de 2017.

36. Urra P. Nuestro reto y nuestra responsabilidad es preservar y continuar desarrollando el sistema de información científica y técnica en salud. ACIMED. 2008;18(4). Disponible en: http:/ / scielo.sld.cu/scielo. php? script $=$ sci_abstract \& pid $=$ S1024-94352008001000001\&lng=es\&nrm= iso\&tlng=es Acceso el 10 de marzo de 2017.

37. Information and Knowledge Societies for Sustainable Development Goals. Champions. Action Line C7 e-Health. Infomed: an innovating, socializing project at the service of Cuban public health. Disponible en: http:/ / www.itu.int/net4/ wsis/prizes/2017/ Acceso el 21 de octubre de 2017.

38. Organización Panamericana de la Salud. Revisión de estándares de interoperabilidad para la eSalud en Latinoamérica y el Caribe. Washington, DC: OPS; 2016. Disponible en: http://iris.paho.org/ xmlui/handle/123456789/28188 Acceso el 10 de marzo de 2017.

Manuscrito recibido el 27 de abril de 2017. Aceptado para publicación, tras revisión, el 9 de noviembre de 2017.
ABSTRACT

\section{Application of eHealth in the Cuban context}

Keywords
The achievements and challenges arising from the application of eHealth in the Cuban context to support universal health are presented. The Ministry of Public Health defined the Computerization Strategy, whose lines of work are based on the components declared in the Strategy and Plan of Action on eHealth of the Pan American Health Organization, and in the Policy for the Computerization of the Society, approved by the Cuban Government. The paper describes the actions of the Cuban strategy elaborated to solve the limitations in the technological infrastructure, to develop the platforms, the tools and the applications required by the health services, and to stimulate the professionals' abilities to use them. The results of eHealth in Cuba are based on the application and evaluation of the strategy implemented in the framework of the improvement of the health sector through projects such as the electronic medical record called Galen Clínicas, the training of human resources and the resources for information and education from the Infomed Health Telematics Network. These models of solidarity and collaborative networking have contributed to universal health and positively impacted on the health system beyond the borders. The challenges of eHealth are the development of initiatives in mobile health - in which there is little experience-; telemedicine — a project that is stopped-; integration and interoperability among applications; the sustainability of the implemented solutions; insufficient technological infrastructure; and strengthening of the legal framework.

Electronic health records; telemedicine; universal coverage; information technologies and communication projects; Cuba. 
RESUMO

\section{Aplicação da eSaúde no contexto cubano}

Palavras-chave Registros eletrônicos de saúde; telemedicina; cobertura universal; projetos de tecnologias de informação e comunicação; Cuba.

As descobertas e desafios decorrentes da aplicação da eSaúde no contexto cubano para apoiar a saúde universal são expostos. O Ministério da Saúde Pública definiu a Estratégia de informatização, cujas linhas de trabalho são baseadas nos componentes declarados na Estratégia e Plano de Ação da eSaúde da Organização Pan-Americana da Saúde e na Política de Informatização da Sociedade aprovada pelo governo cubano. $\mathrm{O}$ artigo especifica as ações da estratégia cubana para resolver as limitações da infraestrutura tecnológica, desenvolver plataformas, ferramentas e aplicações que exigem serviços de saúde, e estimular nos profissionais as habilidades para usá-los. Os resultados da eSaúde em Cuba são o produto da aplicação e avaliação da estratégia aplicada no âmbito da melhoria do setor da saúde, através de projetos como o registro médico eletrônico chamado Galen Clínicas, a preparação do capital humano e os recursos de informação e educação a distância da Rede Telémática de Saúde Infomed. Estes modelos de solidariedade e trabalho colaborativo em redes contribuíram para a saúde universal e impactaram positivamente no sistema de saúde, além das fronteiras. Os desafios da eSaúde consistem no desenvolvimento de iniciativas em saúde móvel, em que há pouca experiência; telemedicina, cujo projeto foi interrompido; a integração e interoperabilidade das aplicações; a sustentabilidade das soluções implementadas; a infra-estrutura tecnológica insuficiente; e o fortalecimento do quadro legal.

gias de informação e comunicação; Cuba.

